\title{
Passivation of Grid-Following VSCs: A Comparison Between Active Damping and Multi-sampled PWM
}

This paper was downloaded from TechRxiv (https://www.techrxiv.org).

\section{LICENSE}

CC BY 4.0

SUBMISSION DATE / POSTED DATE

02-03-2022 / 03-03-2022

\section{CITATION}

Petric, Ivan; Mattavelli, Paolo; Buso, Simone (2022): Passivation of Grid-Following VSCs: A Comparison Between Active Damping and Multi-sampled PWM. TechRxiv. Preprint. https://doi.org/10.36227/techrxiv.19288193.v1

$\mathrm{DOI}$

10.36227/techrxiv.19288193.v1 


\title{
Passivation of Grid-Following VSCs: A Comparison Between Active Damping and Multi-sampled PWM
}

\author{
Ivan Z. Petric, Student Member, IEEE, Paolo Mattavelli, Fellow, IEEE, and Simone Buso, Member, IEEE
}

\begin{abstract}
This paper compares different strategies used to enhance stability properties of grid-following voltage-source converters (VSCs). The focus is on high-frequency properties and performance is examined even above the Nyquist frequency. Due to digital delays, VSC admittance exhibits a non-passive zone above a certain frequency, which introduces negative damping and may destabilize the grid-connected operation. It is shown that typically used active damping (AD) strategies only bring positive impact up to a certain frequency, while deteriorating admittance properties in the high-frequency range. Multisampled pulsewidth modulation (MS-PWM) greatly extends the passive admittance region, using a single-stage current-control loop. Experimental admittance measurements are performed on a single-phase VSC, up to twice the switching frequency. Subsequently, different grid-connected scenarios are tested to show that MS-PWM retains stable operation where AD methods cause instability. In the end, noise propagation is measured and results confirm that, while AD does not bring high sensitivity, MS-PWM offers additional noise suppression. The results of this paper clearly show that MS-PWM is a better candidate for guaranteeing stability of grid-connected VSCs.
\end{abstract}

Index Terms-Active damping (AD), voltage-source converters (VSCs), multi-sampled control (MS-PWM), passivity.

\section{INTRODUCTION}

G RID-FOLLOWING voltage source converters (VSCs) are becoming increasingly present in electric energy distribution grids [1], [2]. However, connecting an activelycontrolled VSC to a grid may bring stability issues [3]. One of such problems may arise when poorly-damped grid resonances get destabilized due to current-loop dynamics of the connecting VSC [3]. This paper focuses on the investigation of harmonic instability at high frequencies, which is enabled by delays of the current control system [4]. In digital control applications, delays arise due to analog-to-digital conversion (ADC), algorithm computation, and modulation process [5]. To prevent the harmonic instability, passivity-based control (PBC) has gained significant attention [4], [6]-[23]. Starting from impedance-based stability assessment [24]-[27], PBC provides a sufficient condition for stability by rendering the VSC input admittance passive, i.e. making its real part nonnegative. Initially, authors focused on passivizing the admittance up to the Nyquist frequency (NF). However, more recently, it has been pointed out that passivity should be investigated also above the NF [8], [28], [29].

The focus of this paper is placed on the most-widely used VSCs that operate using pulse-width modulation (PWM). For uniformly-sampled PWM (US-PWM) [5] with one step computation delay, VSC admittance exhibits negative conductance above one sixth of the sampling frequency $f_{s}$ [4]. For enhancing the passivity, many different strategies of active damping (AD) have been developed. First set of AD methods relies on compensating digital delays by adding phase-leading filters to the controller structure [9], [30]. These methods are simply implemented using a single-stage current control loop; however, they only offer a positive impact at medium frequencies, while the performance is deteriorated close to the NF. The second set relies on decoupling the current loop dynamics from the grid disturbance, by adding additional feedforward or feedback actions to the control stage [4], [10]-[23]. In case of VSCs with $L$ filters, this is achieved by including a derivativebased feedforward of the voltage at the point of common coupling (PCC). The first method that achieves passivity up to the NF is reported in [4] and requires analog or oversampled implementation of the derivative action. This method will be referred to as Derivative Active Damping (D-AD). Several other publications [19]-[23] generalize D-AD approach and extend it to applications with $L C L$ filters, while the underlying principle remains the same. Implementation of $\mathrm{D}-\mathrm{AD}$ without resorting to oversampled derivative is investigated in [10]. This approach will be referred to as Discretized Derivative Active Damping (DD-AD). DD-AD is simply implemented; however, it renders the admittance passive only up to approximately $\frac{f_{s}}{3}$. Reported AD methods, in the best case, passivize the admittance up to the NF for US-PWM; hence, below the switching frequency. In this paper, it is shown that $\mathrm{AD}$ methods have a negative impact at higher frequencies. An alternative approach to US-PWM is the multi-sampled PWM (MS-PWM), where the control action is executed at a rate higher than double the switching frequency [31]-[42]. MSPWM reduces control update and modulation delays, and in this way approaches the naturally-sampled PWM [43]. In [42], it is shown that MS-PWM inherently renders the admittance passive in a wide frequency range.

This paper offers a comparison between AD and MSPWM. Admittance properties are analytically investigated and experimentally validated up to twice the switching frequency. The results show a strong detrimental impact of $\mathrm{AD}$ at high frequencies. MS-PWM, on the contrary, achieves passivity in a wide frequency range, although there are frequency points where the conductance is just below 0. Subsequently, gridconnected operation with anti-resonances formed at different frequencies $f_{\text {res }}$ is experimentally tested. It is shown that both D-AD and DD-AD cause instability for specific values of $f_{\text {res }}$, for which the system without AD is stable. MS-PWM, instead, enables stable operation for all tested cases. In the end, noise sensitivity is examined. Measurements show that AD is not highly sensitive, due to relatively low derivative gain. Still, 

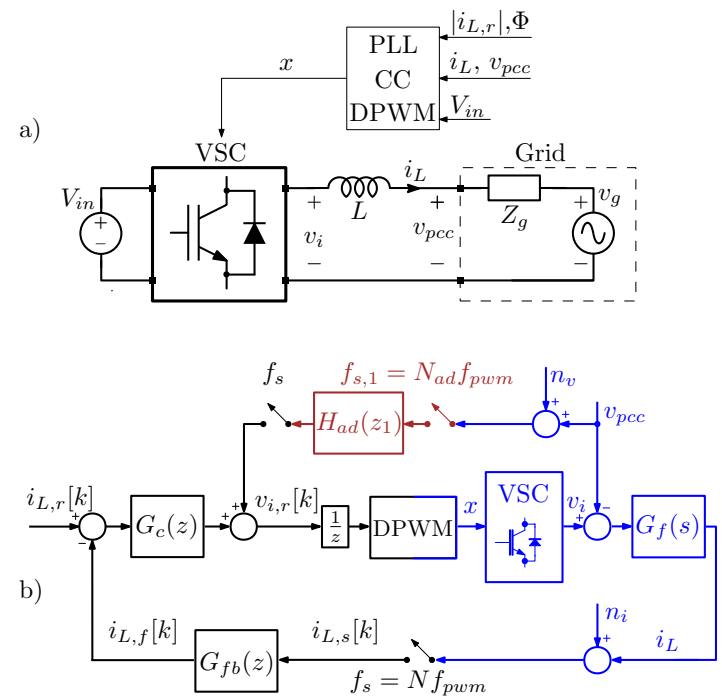

Fig. 1: Illustration of a grid-following VSC: a) schematic representing connection to a grid; b) multi-rate current control system with a feedforward action. Black color depicts blocks executed at current sampling rate. Red color depicts feedforward execution rate. Continuous-time domain is shown in blue. Signals $n_{i}$ and $n_{v}$ represent measurement noise for current and voltage sensing, respectively.

MS-PWM offers additional noise suppression.

The paper is organized as follows: section II describes the current control system of a grid-following VSC. Section III explains the impedance-based stability analysis and admittance passivity. Section IV analyzes strategies for passivity enhancement and shows corresponding experimental admittance measurements. Section V analyzes implications of connecting a VSC with a non-passive admittance to a grid. Experimental tests are provided to show that $\mathrm{AD}$ strategies cause instability for certain grid impedances. Section VI shows results of noise propagation measurements. The conclusions are drawn in Section VII.

\section{CONTROL OF GRID-FOLlOWING VSCS}

Grid-following VSCs are employed in power systems as current-controlled converters. As the most general case, this paper considers a single-phase VSC with an inductive output filter, as shown in Fig. 1 a). However, the analysis is directly applicable to three-phase VSCs in stationary coordinates as well as to any output filter. The current reference $i_{L, r}$ is provided by outer loops that control the required power flow by changing the reference current magnitude $\left|i_{L, r}\right|$ and phase angle $\Phi$, with respect to the voltage at PCC, $v_{p c c}$. Phase-locked loop (PLL) is used to track the fundamental component of $v_{p c c}$. Current controller (CC) processes the error between $i_{L, r}$ and the measured inductor current $i_{L}$. It may also include an action based on $v_{p c c}$, which will be referred to as the Active Damping (AD). Appropriate implementation of AD, which may enhance stability [4], will be analyzed further on. The controller output, i.e. the reference voltage $v_{i, r}$, is processed by the digital pulse-width modulator (DPWM), which outputs the transistor switching signal $x$ with the duty cycle $D$. The switching frequency is $f_{p w m}=\frac{1}{T_{p w m}}$. Input dc bus voltage $V_{i n}$ is used for $v_{i, r}$ scaling [5].

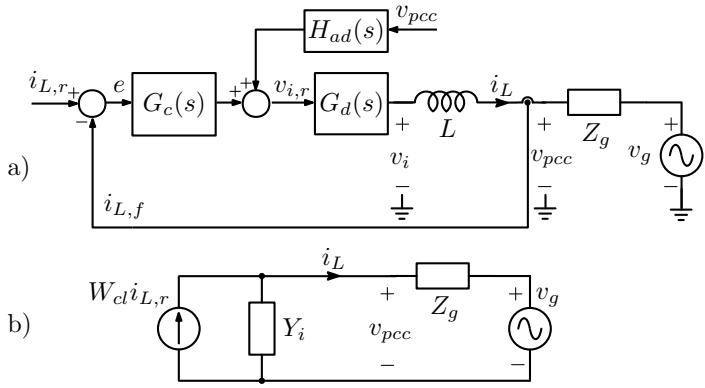

Fig. 2: s-domain representation of a grid-following VSC: a) current control system and grid connection; b) Norton equivalent circuit used for impedancebased stability assessment.

Closed-loop block diagram of the current control system is shown in Fig. $1 \mathrm{~b}$ ), where $v_{p c c}$ is shown as a disturbance. Difference between the VSC output voltage $v_{i}$ and $v_{p c c}$ is applied to the output inductive filter:

$$
G_{f}(s)=\frac{i_{L}(s)}{v_{i}(s)-v_{p c c}(s)}=\frac{1}{s L},
$$

where $s$ is the complex variable of the Laplace transform and $L$ is the inductance value. The resulting current $i_{L}$ is sampled by an analog-to-digital converter (ADC) with a rate $f_{s}=N f_{\text {pwm }}$, where $N$ is the multisampling (oversampling) factor. The frequency $f_{s}$ will be referred to as the sampling and control frequency, and its inverse $T_{s}=\frac{1}{f_{s}}$ as the control period. Values $N=\{1,2\}$ correspond to uniformly singlesampled (SS-PWM) and double-sampled (DS-PWM) control [5], respectively. For $N>2$, multi-sampled PWM (MS-PWM) control loop is formed [32]. The control frequency determines the execution rate of feedback filter $G_{f b}(z)$ and controller $G_{c}(z)$, where $z$ is the Z-domain variable corresponding to $T_{s}$. Due to finite algorithm computation time, the controller output is often delayed by one control period [5]. The control system in Fig 1 b) also includes the AD block $H_{a d}\left(z_{1}\right)$ [4]. The execution rate of the $\mathrm{AD}$ block is independently chosen as $f_{s, 1}=N_{a d} f_{p w m}$, where $N_{a d}$ will be referred to as the $\mathrm{AD}$ oversampling factor. The $\mathrm{Z}$-domain variable $z_{1}$ corresponds to $T_{s, 1}=\frac{1}{f_{s, 1}}$. In case $f_{s, 1} \neq f_{s}$, a rate conversion needs to be included after $H_{a d}$.

\section{A. S-domain representation}

In this paper, modeling is performed in s-domain to allow investigation of VSC admittance properties even above the NF [8]. The s-domain representation of the system shown in Fig $1 \mathrm{~b}$ ), without including the feedback filter, is shown in Fig 2 a). All time delays are represented by a unique block $G_{d}(s)$.

The triangular carrier is chosen for the DPWM [5]. Its small-signal model is obtained using describing function approach [44]:

$$
G_{d p w m}(s)=\frac{d(s)}{m(s)}=\frac{1}{2}\left(e^{-s D T_{s}}+e^{-s(1-D) T_{s}}\right),
$$

where $m$ is the modulating waveform, equal to $v_{i, r}$ after one step delay, and $d$ is the continuous-time duty-cycle of $x$, used in averaged modeling [5]. From (2), it is clear that digitallycontrolled VSCs exhibit operating point dependency, which 
is often overlooked when using delay or $\mathrm{ZOH}$ models for the DPWM, due to its limited impact at low and medium frequencies [45]. However, in [45], it is shown that this nonlinearity may yield significant impacts in the high frequency range. For $D=0.5$, DPWM model reduces to a pure time delay $e^{-s \frac{T_{s}}{2}}$. As this $D$ is the mean value of duty-cycles for sinusoidal ac operation, it will be used for the subsequent analysis. Together with one step delay added due to algorithm execution time [5], the total time delay in digital current control system is equal to:

$$
G_{d}(s)=\frac{v_{i}(s)}{v_{i, r}(s)} \approx e^{-s \tau_{D}}=e^{-s \frac{3}{2} T_{s}}=e^{-s \frac{3}{2} \frac{T_{p w m}}{N}}
$$

As a standard approach for sinusoidal reference tracking, the proportional-resonant (PR) controller is used in this paper [5]:

$$
G_{c}(s)=k_{p}+k_{r} \frac{s}{s^{2}+\omega_{1}^{2}},
$$

where $k_{p}$ and $k_{r}$ are the proportional and resonant gains, respectively, and $\omega_{1}$ is the angular frequency of the current reference. As an example considered in this paper, the proportional gain is chosen as $k_{p}=\omega_{c} L$, where $\omega_{c}$ is the sought angular crossover frequency of the current loop [5]. The resonant gain is chosen as $k_{r}=\frac{1}{10} \omega_{c} k_{p}$, which limits its impact to lower frequencies [5]. The value of $\omega_{c}$ relative to the angular switching frequency $\omega_{\text {pwm }}$, labeled as $\alpha=\frac{\omega_{c}}{\omega_{p w m}}$, will be referred to as the relative bandwidth. Value of $\alpha$ is limited by system delays and PWM carrier frequency [5], [46].

\section{IMPEDANCE-BASED STABILITY}

\section{A. Norton equivalent circuit and admittance passivity}

The impedance-based stability assessment requires representing the VSC with its Norton equivalent circuit, as in Fig $2 \mathrm{~b})$. The Norton current source determines the short circuit current of the VSC, i.e. the value of $i_{L}$ when $v_{p c c}=0$ :

$$
W_{c l}(s)=\left.\frac{i_{L}(s)}{i_{L, r}(s)}\right|_{v_{p c c}=0}=\frac{W_{o l}(s)}{1+W_{o l}(s)},
$$

where $W_{o l}(s)=G_{c}(s) G_{d}(s) G_{f}(s)$ is the open-loop gain of the corresponding system. The input admittance $Y_{i}$ is found as the inductor current response to the voltage $v_{p c c}$, when current reference is set to 0 :

$$
Y_{i}(s)=-\left.\frac{i_{L}(s)}{v_{p c c}(s)}\right|_{i_{L, r}=0}=\frac{G_{f}(s)\left(1-H_{a d}(s) G_{d}(s)\right)}{1+W_{o l}(s)} .
$$

From (6), it can be seen that the VSC input admittance is shaped by the output filter and the current control system, which includes digital delays and, if implemented, AD. The impact of outer loops on admittance is not modeled, as this paper focuses on properties at frequencies significantly above their bandwidth [7].

Considering the VSC connection to a non-ideal grid, i.e. $Z_{g} \neq 0$, the inductor current can be calculated using the equivalent circuit parameters as:

$$
i_{L}(s)=\frac{W_{c l}(s)}{1+Y_{i}(s) Z_{g}(s)} i_{L, r}(s)-\frac{Y_{i}(s)}{1+Y_{i}(s) Z_{g}(s)} v_{g}(s) .
$$

Assuming that $W_{c l}$ is stable, the stability of the gridconnected VSC depends on the product $Y_{i}(s) Z_{g}(s)$, which is referred to as the minor-loop gain [25]. Stability analysis can be performed by applying the Nyquist stability criterion to the minor-loop gain ${ }^{1}$.

The input admittance $Y_{i}$ is said to be passive if its phase is in the range $\left(-\frac{\pi}{2}, \frac{\pi}{2}\right)$, i.e. if its real part is greater or equal than 0 [7]. Imposing the requirement for passive $Y_{i}$, such as in [6], arises from the fact that if both $Y_{i}$ and $Z_{g}$ are passive, the phase of their product never crosses $\pm \pi$. Therefore, minorloop gain always satisfies the Nyquist criterion.

\section{B. Impact of current control on passivity}

Considering a system without $\mathrm{AD}$, the real part of $Y_{i}$ is calculated for a proportional controller [4]:

$$
\begin{aligned}
& \left.\operatorname{Re}\left\{Y_{i}(j \omega)\right\}\right|_{H_{a d}=0, k_{r}=0}= \\
& =\frac{\omega_{c}}{L} \frac{\cos \left(\omega \tau_{D}\right)}{\left(\omega_{c}-\omega \sin \left(\omega \tau_{D}\right)\right)^{2}+\left(\omega \cos \left(\omega \tau_{D}\right)\right)^{2}},
\end{aligned}
$$

where $\omega$ is the angular frequency. From (8), it is clear that delays cause the input admittance to feature negative real part at frequencies where the cosine term is negative. As analyzed in [42], the peak value of negative conductance is increased for higher values of $\omega_{c}$ and $\tau_{D}$, as well as for lower values of $L$. The expression (8) neglects $k_{r}$ because its impact is negligible at high frequencies, where $\operatorname{Re}\left\{Y_{i}\right\}$ turns negative [42].

\section{Experimental setup and admittance measurements for DS- $P W M$ without $A D$}

For experimental admittance measurements and subsequent grid-connected tests, an industrial full-bridge VSC, described in Table I, is used. The VSC is operated using the bipolar modulation. Input voltage is provided by regenerative power system Keysight RP7962A.

TABLE I: VSC and control parameters

\begin{tabular}{llll}
\hline \hline Description & label & value & unit \\
\hline Nominal power & $S_{n}$ & 3 & $\mathrm{kVA}$ \\
Nominal input voltage & $V_{i n}$ & 400 & $\mathrm{~V}$ \\
Nominal PCC voltage & $v_{p c c, R M S}$ & 230 & $\mathrm{~V}$ \\
Fundamental frequency & $f_{1}$ & 50 & $\mathrm{~Hz}$ \\
Switching frequency & $f_{p w m}$ & 20 & $\mathrm{kHz}$ \\
Filter inductance & $L$ & $\{1.5,2.5\}$ & $\mathrm{mH}$ \\
Dead time & $t_{d t}$ & 500 & $\mathrm{~ns}$ \\
\hline \hline
\end{tabular}

${ }^{1}$ The admittance $Y_{i}$ in (6) is the so-called single-frequency model [8], [45]. For digitally controlled VSCs, sampling and PWM create sideband components that can be taken into account using multi-frequency modeling [8]. The impact of sidebands is limited [8] and is mainly evident at very high frequencies. It is important to note that the multi-frequency model is by itself non-linear; hence, using control tools derived for linear systems, such as the Nyquist stability criterion, is not strictly accurate. However, it will be shown that experimentally measured admittances are in a good match with the corresponding single-frequency $Y_{i}$ in the frequency range of interest for stability verification. 


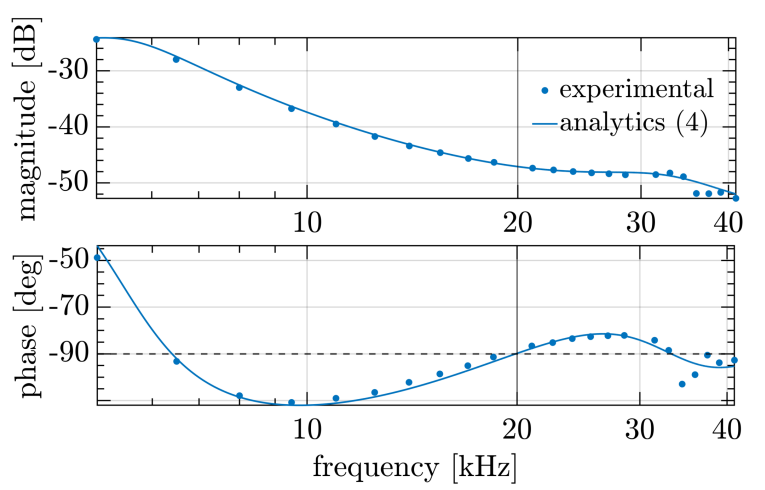

Fig. 3: Admittance measurements for the case of DS-PWM without AD: $N=$ $2, H_{a d}=0, L=1.5 \mathrm{mH}, \alpha=0.2$. The vertical line marks the switching and the Nyquist frequency.

The control system is implemented on an NI sbRIO-9606, which is based on a Xilinix Zynq 7020 all programmable system on chip (AP-SoC). The controller is discretized using the impulse-invariant approach [5]. For all subsequent admittance measurements, $L=1.5 \mathrm{mH}$ and $\alpha=0.2$. For strategies other than MS-PWM, current is sampled and the control algorithm is executed twice per switching period, i.e. DS-PWM is used. Sampling instants coincide with peak and valley of the carrier, which results in average current sampling [5].

Input admittance $Y_{i}$ is measured by injecting voltage perturbation components at PCC and measuring the current response, as in [42]. The perturbation voltage is generated using a power operational amplifier MP118 from APEX. Measurements are performed for 23 frequencies, one at a time, from $5 \mathrm{kHz}$ to $41 \mathrm{kHz}$, which is just above $f_{s}$ for $N=2$. Perturbation voltage is calculated to obtain, at least, $100 \mathrm{~mA}$ of perturbation component of $i_{L}$, to provide a good measurement resolution. Tektronix 5 series oscilloscope is used to acquire $i_{L}$ and $v_{p c c}$ with data length equal to $40 \mathrm{~ms}$ and sampling rate of 250 MS/s. Data is postprocessed in MATLAB, by calculating the FFT of $-i_{L}$ and $v_{p c c}$ and determining the admittance based on their ratio at the perturbation frequency. Same measurement approach, described in more details, is used in [42]. All admittance measurements shown in this paper were conducted for $v_{g}=0$, leaving only perturbation voltage component at PCC. This sets the steady-state operating point to $D=0.5$, which is compliant with the previous analysis. Measuring the admittance while the VSC is connected to a non-zero voltage ac grid would result in a sinusoidal operating point with fundamental frequency; hence, for an operating point dependent system, results would not be compliant with the small-signal analysis. Note that these measurements were conducted, and the results were found to be somewhere in between dc results for all operating points in the sinusoidal ac range. More considerations on this can be found in [42], [45].

As a benchmark, admittance measurements are given for the case of DS-PWM without AD. These results are shown in Fig. 3 where it can be seen that a very good match with analytics is obtained, even above the NF. Some mismatch is seen close to $f_{s}$. Using the analytical approach from [45], where sampling sidebands are taken into account, near-perfect

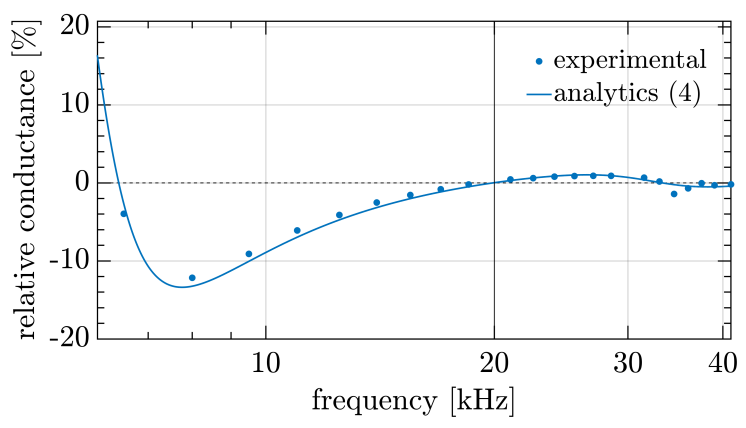

Fig. 4: Relative conductance for the case of DS-PWM without AD: $N=2$, $H_{a d}=0, L=1.5 \mathrm{mH}, \alpha=0.2$. The vertical line marks the switching and the Nyquist frequency.

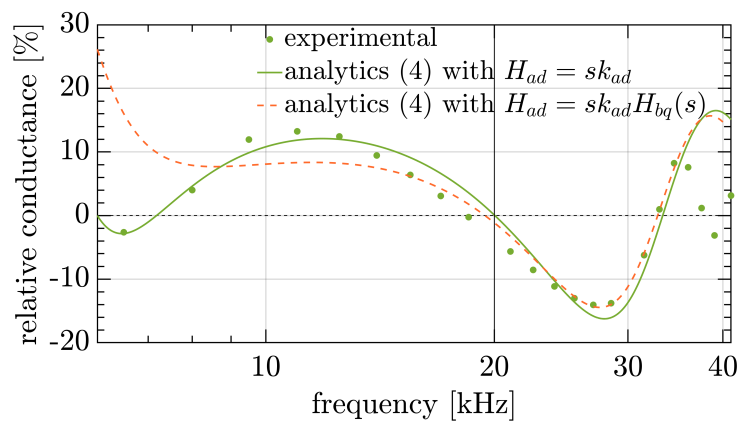

Fig. 5: Relative conductance for the case of D-AD: $N=2, N_{a d}=16$, $H_{a d}=\frac{k_{a d}}{T_{s, 1}}\left(1-z_{1}^{-1}\right), L=1.5 \mathrm{mH}, \alpha=0.2$. The vertical line marks the switching and the Nyquist frequency. Yellow dashed line shows the impact of including an additional filter $H_{b q}$ in cascade with the derivative gain [4].

match is obtained up to $2 f_{s}$. Note the existence of two nonpassive zones, where phase drops below -90 degrees. The first one starts at approximately $6.5 \mathrm{kHz}$, which is consistent with the often mentioned critical frequency $f_{\text {crit }}=\frac{f_{s}}{6}$, for which the cosine term in (8) changes sign [4]. Another narrow non-passive zone appears above the NF. Goal of the passivitybased design, which is analyzed in the following section, is to suppress these non-passive zones. As an additional illustration, the real part of measured $Y_{i}$, relative to the nominal VSC admittance $Y_{n}=\frac{S_{n}}{U_{n}^{2}}=56.7 \mathrm{mS}$, is shown in Fig. 4 .

\section{ANALYSIS AND EXPERIMENTAL VERIFICATION OF PASSIVITY-ENHANCEMENT STRATEGIES}

\section{A. Active damping}

First method used for comparison is D-AD [4], which achieves passivity up to the NF by adding a derivative feedforward of $v_{p c c}$ to the controller output, $H_{a d}(s)=s k_{a d}$. Calculating the real part of (6) for a proportional controller, as in [4], yields the same expression as (8) multiplied by $\left(1-k_{a d} \frac{\omega^{2}}{\omega_{c}}\right)$. The active damping gain $k_{a d}$ is chosen such that the added term changes sign at the same frequency as the cosine term [4], i.e.:

$$
k_{a d}=\frac{4 \tau_{D}^{2}}{\pi^{2}} \omega_{c}
$$

For all subsequent tests employing $\mathrm{AD}$, gain $k_{a d}$ is calculated using (9). In [4], it is shown that the derivative 
action must be implemented in analog domain, or using oversampling. The latter approach is chosen in this paper, in order to keep the implementation fully digital. BackwardEuler discretization is chosen, as advised in [4]. Referring to Fig. 1 b), D-AD is implemented with $N=2, N_{a d}=16$, $H_{a d}\left(z_{1}\right)=k_{a d} f_{s, 1}\left(1-z_{1}^{-1}\right)$. AD oversampling factor was chosen to be as high as allowed by the control platform.

Relative value of measured conductance is shown in Fig. 5. A very good match with s-domain analytics is obtained, apart for frequencies very close to $f_{s}$. It is assumed that this mismatch comes from not including the sampling effects. Results in Fig. 5 confirm that D-AD is capable of improving passivity up to the $\mathrm{NF}^{2}$. It can be seen that, above the NF, a non-passive zone is introduced with a peak of negative conductance being slightly higher than for the case of DSPWM without AD. In [12], this effect is mentioned, however it is not thoroughly investigated in the literature, even if, from [8], it is clear that admittance properties above the NF need to be examined. D-AD was also tested for other values of $k_{a d}$; however, these results are not shown due to paper length limitations. The trend is that higher values of $k_{a d}$ add more positive damping in the frequency region from $\frac{f_{s}}{6}$ to the NF and more negative damping in the region above the $\mathrm{NF}$, where the peak negative conductance is found. Lower values of $k_{a d}$ decrease the peak negative conductance above the NF; however, passivity is not achieved around $\frac{f_{s}}{6}$. It is interesting to note that measurements were also conducted for other steady-state values of $D$. It is found that, as $D$ approaches extreme values, the non-passive zone above the NF becomes less emphasized. Therefore, for sinusoidal ac operation, highest introduced negative damping is expected around zero crossings of $v_{g}$, where $D=0.5$.

Second compared AD method, DD-AD, uses the same derivative action as $\mathrm{D}-\mathrm{AD}$, however, without oversampling: $N=2, N_{a d}=2, H_{a d}(z)=k_{a d} f_{s}\left(1-z^{-1}\right)$. DD-AD is applied in [10], with a specific parameter design procedure. However, it was verified that implementing derivative gain as (9) brings practically the same results, without needing to use one fixed value of bandwidth as in [10]. DD-AD is not easily modeled at frequencies close to and above the NF due to derivative action behavior. In [10], Z-domain modeling is used, which yields significant mismatch as frequencies approach the NF. Hence, in this paper, $H_{a d}$ is directly transformed to s-domain, using $z=e^{s T_{s, 1}}$. Results are reported in Fig. 6. It can be seen that a good match between experiments and analytics is obtained. From Fig. 6, it can be seen that DD-AD improves passivity at medium frequencies; however, above approximately $\frac{f_{s}}{3}$, passivity is lost. The same frequency limit is reported in papers that use single-loop AD, by adding derivative gain to the controller structure [9]. Note that the peak of negative conductance for DD-AD is slightly reduced

\footnotetext{
${ }^{2}$ In [4], an additional biquad filter $H_{b q}$ is added in cascade with the derivative action, which is referred to as the modified active damping (M$\mathrm{D}-\mathrm{AD})$. This filter adds damping to the narrow non-passive zone around $\frac{f_{s}}{6}$ that remains when only derivative $H_{a d}$ is used. It is not included in this paper, as its impact above the NF is negligible. Analytical trace corresponding to M-D-AD is shown in Fig. 5. This trace was validated but measurements are not shown for figure clarity.
}

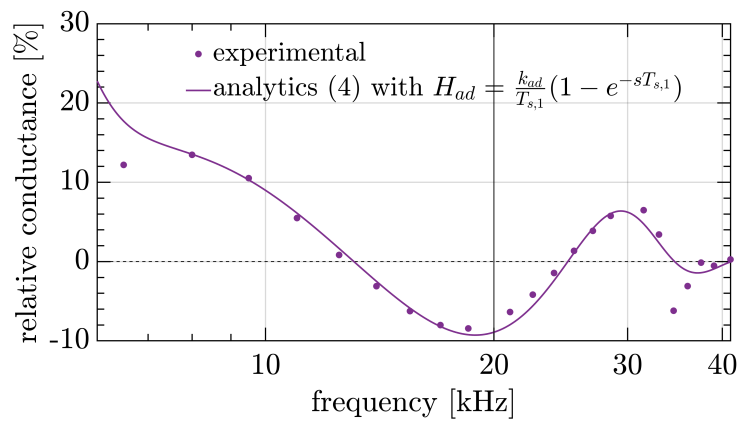

Fig. 6: Relative conductance for the case of DD-AD: $N=2, N_{a d}=2$, $H_{a d}=\frac{k_{a d}}{T_{s, 1}}\left(1-z_{1}^{-1}\right), L=1.5 \mathrm{mH}, \alpha=0.2$. The vertical line marks the switching and the Nyquist frequency.

compared to the case of DS-PWM without AD.

\section{B. Multi-sampled PWM}

It has been recently shown that a suitable candidate for gridconnected converters is the single-stage MS-PWM control, where $N>2$ and $H_{a d}=0$ [42]. Instead of compensating digital delays, MS-PWM relies on reducing them by increasing the update rate of the controller output. Main advantage of MS-PWM, compared to other strategies for DS-PWM delayreduction [47], [48], is that both delays, due to controller output update and digital modulation, are reduced [32]. MSPWM improves dynamic properties and approaches analog, naturally-sampled, PWM [32]. Analysis of MS-PWM operation and nonlinearities that may arise can be found in [41]. With MS-PWM, switching ripple is introduced in the feedback. While filtering the switching ripple is beneficial regarding modulator linearity [37], it strongly deteriorates dynamic improvements. Analysis of MS-PWM operation without ripple filtering can be found in [32], [41] and such an approach is considered in this manuscript as well.

In [42], a detailed investigation of MS-PWM impact on admittance properties is given, for many values of $N$. There, it is shown that MS-PWM expands the passive region of $Y_{i}$, without needing to resort to AD. For lower values of $N$, such as 4 , a small non-passive zone appears close to $f_{p w m}$; however, negative damping is greatly reduced compared to $N=2$. Further increase of $N$ strongly reduces the non-passive zone so that fully passive admittance is measured up to $1.5 f_{\text {pwm }}$ for $N>8$ in [42]. Oversampling factor $N$ is limited by the available processing power with respect to the switching frequency. As an example used for this paper, admittance is measured for $N=16$. In the following section, $N=4$ is also implemented to show that it is sufficient to retain the stability of the tested grid-connected operation. For the case of MSPWM, the measured conductance is shown in Fig. 7. It can be seen that $Y_{i}$ is almost fully passive, except for a very narrow range, where the conductance is just below 0 .

\section{Comparison of effectiveness}

In Fig. 8, conductances for all tested cases are shown in an extended frequency range. As mentioned in the previous 


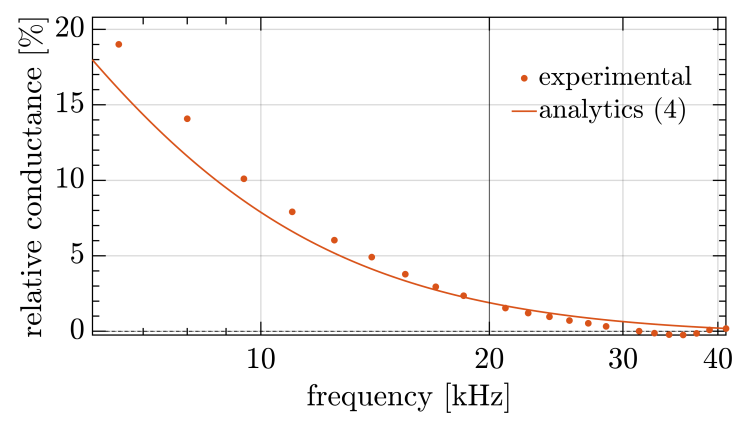

Fig. 7: Relative conductance for the case of MS-PWM: $N=16, H_{a d}=0$, $L=1.5 \mathrm{mH}, \alpha=0.2$. The vertical line marks the switching frequency.

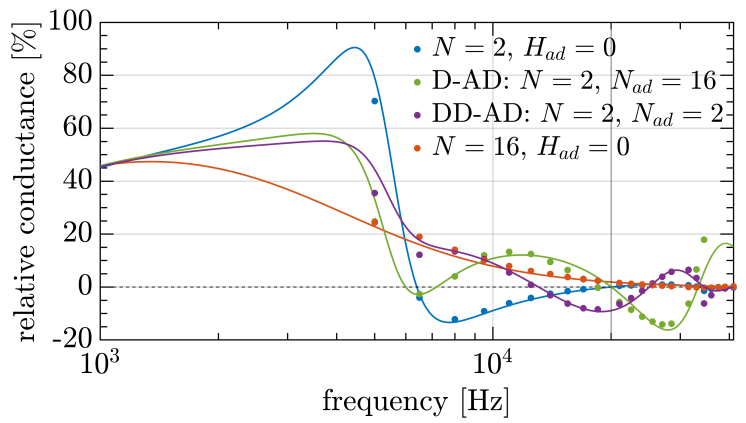

Fig. 8: Comparison of conductances for all tested control strategies: $L=1.5$ $\mathrm{mH}, \alpha=0.2$. The vertical line marks the switching frequency.

subsection, the narrow non-passive zone around $6.5 \mathrm{kHz}$ for $\mathrm{D}-\mathrm{AD}$ can be removed using the biquad filter from [4]. It can be seen that both D-AD and DD-AD are able to extend the passive zone; however, for both methods, another emphasized non-passive zone is introduced, which is not present for the case of DS-PWM without AD. This points to the fact that the addition of $\mathrm{AD}$ only shifts the non-passive zone to higher frequencies and can, therefore, compromise stability, instead of guaranteeing it. It is important to note that these non-passive zones are found in the frequency range where single-frequency model shows a good match with measurements. This is important as it can be assumed that assessing stability using the Nyquist criterion most-likely yields valid conclusions. MSPWM shows a natural drop towards zero conductance, where $Y_{i}$ starts to behave as an inductor. The admittance is measured for other values of $N$ as well, and similar trends are obtained. As $N$ is increased, the non-passive frequency range and peak negative damping are strongly reduced compared to DS-PWM without $\mathrm{AD}, \mathrm{D}-\mathrm{AD}$, and $\mathrm{DD}-\mathrm{AD}$. The summary of measured conductances is shown in Table II, by providing peaks of negative values and frequencies for which they are found. Results are also given for a second inductor with $L=2.5$ $\mathrm{mH}$. For MS-PWM and $N=16$, analytics predict the nonpassive zone to fall outside of the measurement range.

\section{PASSIVITY IMPLICATIONS IN GRID-CONNECTED SCENARIOS}

Impedance-based analysis from subsection III.B predicts that a VSC can be destabilized if connected to a grid with
TABLE II: Comparison of conductance minimums and frequencies for which they are found: $\min \left\{\mathrm{R}_{\mathrm{e}}\left\{\mathrm{Y}_{\mathrm{i}}\right\}\right\}[\%] @ f_{\min }[\mathrm{kHz}]$.

\begin{tabular}{|c|c|c|c|c|}
\hline \multirow{2}{*}{$\begin{array}{l}\text { PWM } \\
\mathrm{AD}\end{array}$} & \multicolumn{3}{|c|}{ DS-PWM } & \multirow{2}{*}{$\begin{array}{l}\text { MS-PWM } \\
\text { w/o AD }\end{array}$} \\
\hline & w/o AD & D-AD & DD-AD & \\
\hline \multicolumn{5}{|c|}{$L=1.5 \mathrm{mH}$} \\
\hline exp. & $-12.2 @ 8$ & -14.1@27 & $-8.4 @ 18.5$ & $-0.2 @ 36$ \\
\hline analyt. & $-13.4 @ 7.8$ & $-16.2 @ 27.9$ & $-9.3 @ 18.9$ & $-0.1 @ 81$ \\
\hline \multicolumn{5}{|c|}{$L=2.5 \mathrm{mH}$} \\
\hline exp. & $-8.2 @ 8$ & $-7.9 @ 27$ & $-5 @ 18.5$ & $-0.1 @ 36$ \\
\hline analyt. & $-8 @ 7.8$ & $-9.7 @ 27.9$ & $-5.6 @ 18.9$ & $-0.05 @ 81$ \\
\hline
\end{tabular}

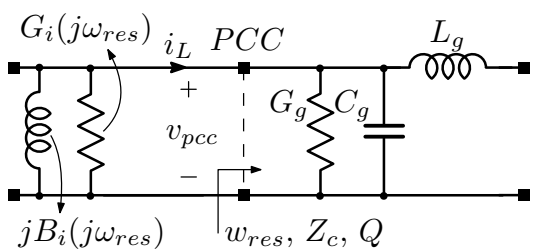

Fig. 9: Impedance network at the frequency of grid anti-resonance. VSC admittance is represented as $Y_{i}\left(j \omega_{r e s}\right)=G_{i}\left(j \omega_{\text {res }}\right)+j B_{i}\left(j \omega_{\text {res }}\right)$. Grid impedance features passive damping, represented by $G_{g}$.

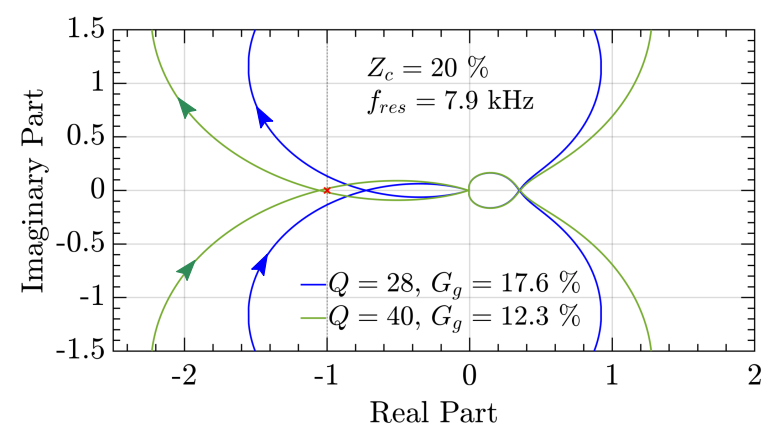

Fig. 10: Nyquist plots of the minor-loop gain $Y_{i}(j \omega) Z_{g}(j \omega)$ for the case of DS-PWM without AD and two different grid anti-resonances illustrated in Fig. 9. Corresponding $Y_{i}$ is shown in Fig. 3 and $G_{i}\left(j \omega_{\text {res }}\right)=-13.3$ $\%$. Blue line shows $Z_{g}$ with sufficient passive damping to compensate the VSC negative conductance. Green line shows $Z_{g}$ that would result in unstable operation.

a certain $Z_{g}$. For grid-following VSCs with inductive filters, a parallel anti-resonance that coincides with the non-passive zone of $Y_{i}$ can cause instability [4]. In this section, operation stability is tested for all strategies compared in section IV, by connecting the VSC to a grid with a specific $Z_{g}$. For all results in this section, $L=1.5 \mathrm{mH}$ and $\alpha=0.2$.

\section{A. Analysis of the equivalent impedance network}

Suppose that the analyzed VSC is connected to a grid with an anti-resonance formed by $L_{g}$ and $C_{g}{ }^{3}$. Grid passive damping can be modeled as a parallel conductance $G_{g}$. Such grid impedance can, therefore, be described using its antiresonant frequency $f_{\text {res }}=\frac{\omega_{\text {res }}}{2 \pi}=\frac{1}{\sqrt{L_{g} C_{g}}}$, characteristic impedance $Z_{c}=\sqrt{\frac{L_{g}}{C_{g}}}$, and quality factor $Q=\frac{1}{Z_{c} G_{g}}$.

${ }^{3}$ Note that specific $L_{g}$ and $C_{g}$ should not be understood as parts of a properly designed $L C L$ filter, but as anti-resonance forming components that may be found due to, for example, other connected VSCs. 
Analysis of passive damping is important, as a non-passive $Y_{i}$ does not necessarily destabilize the grid anti-resonance with realistic values of $Q$ [42].

The equivalent impedance network at the frequency of grid anti-resonance is illustrated in Fig. 9. At $\omega_{\text {res }}$, VSC input admittance is represented by its conductance $G_{i}\left(j \omega_{\text {res }}\right)$ and susceptance $B_{i}\left(j \omega_{\text {res }}\right)$. Intuitively, from Fig. 9, it can be concluded that overall system damping at $\omega_{\text {res }}$ is impacted by the parallel connection of $G_{i}\left(j \omega_{\text {res }}\right)$ and $G_{g}$. Hence, the value of $G_{i}\left(j \omega_{\text {res }}\right)+G_{g}$ indicates the damping of the resonant network. This intuitive approach is valuable as it helps to predict the stability based solely on admittance measurements, without knowing the analytic expression for $Y_{i}$. Formal approach, in case $Y_{i}$ is analytically known, is to analyze stability using Nyquist plots of the minor-loop gain. Examples of Nyquist plots are shown in Fig. 10, for $N=2$ without $\mathrm{AD}$, i.e. $Y_{i}$ from Fig. 3. Plots are given for two anti-resonant networks with $f_{\text {res }}=7.9 \mathrm{kHz}$ and characteristic impedance $Z_{c}=20 \%$, given relative to the nominal VSC impedance. For $Q=28$, Nyquist plot predicts stable connection, while for $Q=40$, instability is predicted. Note that boundary value of $Q$, for which system becomes unstable, coincides with the case $G_{i}\left(j \omega_{r e s}\right)=G_{g}$.

\section{B. Experimental grid-connected tests}

Experimental prototype is the same used for admittance measurements. Grid is formed using Chroma 6460 programmable AC power supply and passive filters to form specific anti-resonances. It was noticed that our Chroma was adding some damping at high frequencies; hence, it was decoupled with an additional $L C$ filter with anti-resonant frequency equal to $650 \mathrm{~Hz}$. This filter does not have direct implications on subsequent tests and its purpose is just to form an almost ideal voltage source at frequencies of interest. Voltage levels are reduced to $V_{i n}=250 \mathrm{~V}$ and $v_{p c c}=110$ V RMS to reduce any impact of unstable operation. The VSC operates as a rectifier with unity power factor, as our Chroma does not support regenerative operation.

Anti-resonant networks that coincide with tested nonpassive zones are formed using an inductor $L_{g}=72 \mathrm{uH}$ and different parallel capacitors $C_{g}$. First anti-resonance, $Z_{g, 1}$, with $f_{\text {res }}=7.9 \mathrm{kHz}$ is obtained with $C_{g}=5.6 \mathrm{uF}$. It is very close to the peak of negative conductance for $N=2$ without AD. Second anti-resonance, $Z_{g, 2}$, with $f_{\text {res }}=18.6$ $\mathrm{kHz}$ is formed using $C_{g}=1 \mathrm{uF}$. It is very close to the peak of negative conductance for DD-AD. Note that this antiresonance is close to $f_{\text {pwm }}$, which results in high ripple of $v_{p c c}$. Third anti-resonance, $Z_{g, 3}$, with $f_{\text {res }}=28.4 \mathrm{kHz}$ is formed using $C_{g}=440 \mathrm{nF}$. It is very close to the peak of negative conductance for D-AD.

Results of all grid-connected tests are shown in Fig. 11. Oscilloscope screen shows $i_{L}$ (orange) and $v_{p c c}$ (red). Each column of subfigures shows results for one grid anti-resonance. In first two rows, results are shown for the case of MS-PWM with $N=4$ and $N=16$, which enable stable operation for all tested anti-resonances. Transient is tested by imposing a step reference change from $4 \mathrm{~A}$ to $15 \mathrm{~A}$, at peak of the fundamental component. Results for $N=4$ are given to show that it is not always necessary to raise $N$ to very high values. Note that, based on results from [42], MS-PWM with $N=4$ results in a small non-passive zone around $f_{\text {pwm }}$, which coincides with the anti-resonance $Z_{g, 2}$. However, introduced negative damping is strongly suppressed; hence, passive grid damping is enough to compensate it. Note that specific values of $Q$ were not measured; however, it was verified that $Z_{g, 1}$ features higher passive damping than the other two.

In the third row of Fig. 11, results are shown for DSPWM strategies that feature non-passive zones, which coincide with the correspondingly tested anti-resonances. In Fig. 11 g), strong resonant oscillations are triggered; however, due to sufficiently high passive damping of $Z_{g, 1}$, instability is prevented. In Figs. $11 \mathrm{~h}$ ) and i), instability occurs for AD strategies. For these anti-resonances, DS-PWM without AD is stable; hence, results show transients when respective AD strategy is activated. In Fig. $11 \mathrm{~h}$ ), overvoltage protection is triggered when DD-AD is activated, and the VSC is turned off. In Fig. 11 i), when D-AD is activated, protection is not triggered; however, instability clearly occurs and the controller output jitters from positive to negative saturation limits. From Fig. 11 i), it is clear that oscillations are much more emphasized around zero crossings of $v_{p c c}$, where $D=0.5$. This is in agreement with comments made in subsection IV.A. that, for $\mathrm{D}-\mathrm{AD}$, the tested non-passive zone has highest negative peak for $D=0.5$. Note that this case corresponds to instability caused by anti-resonance above the NF. Its enabling mechanism is the coupling between sampling and PWM sidebands, which allows the controller to react to a disturbance above the NF [8]. In fourth row of Fig. 11, zoomed waveforms corresponding to the third row are given to show that oscillations are triggered at the anti-resonant frequency.

It can be concluded that the implementation of DS-PWM with $\mathrm{AD}$ may result in unstable operation for a certain grid anti-resonance. Hence, AD does not guarantee stability of a grid-connected VSC for any $Z_{g}$. On the other hand, MS-PWM retains stability for all tested cases.

\section{NOISE SENSITIVITY}

Another question that arises for AD strategies is their noise sensitivity [17], as derivative action is applied to $v_{p c c}$. The goal of this section is to quantify the noise propagation from sensing to the output current $i_{L}$, for passivizing control strategies tested beforehand. As seen in Fig 1 b), AD strategies bring an additional voltage noise path to the system, from sensed $n_{v}$ to $i_{L}$. Detailed analysis of noise propagation for dc-dc converters is given in [40], and the same measurement approach is taken in this paper. Relative bandwidth is reduced to $\alpha=0.1$ to obtain closer phase margins between DS-PWM and MS-PWM, which has an impact on noise propagation [40]. Reference current is set to 5 A RMS and $L=2.5 \mathrm{mH}$ is used in order to capture current signal using oscilloscope with $2 \mathrm{~A} /$ div scale. Larger scale was resulting in noise floor of the oscilloscope compromising $i_{L}$ noise measurements. Output variance, up to $\frac{f_{p w m}}{2}$, is calculated in MATLAB using 200 ms of oscilloscope data sampled with $250 \mathrm{MS} / \mathrm{s}$. Fundamental 
$Z_{g, 1}: f_{\text {res }}=7.9 \mathrm{kHz}, Z_{c}=20 \%$

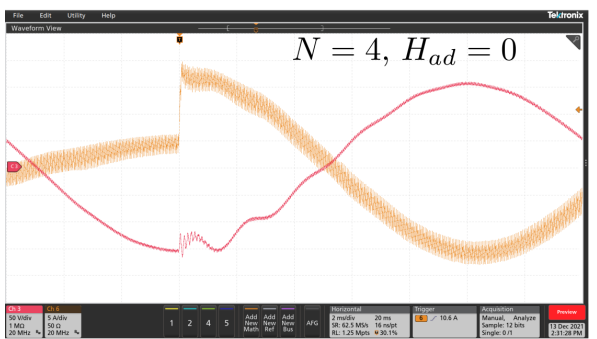

a)
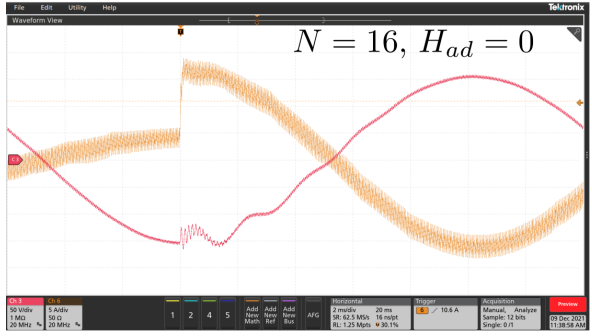

d)

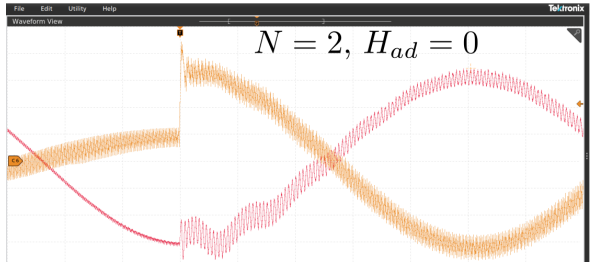

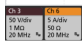

g)

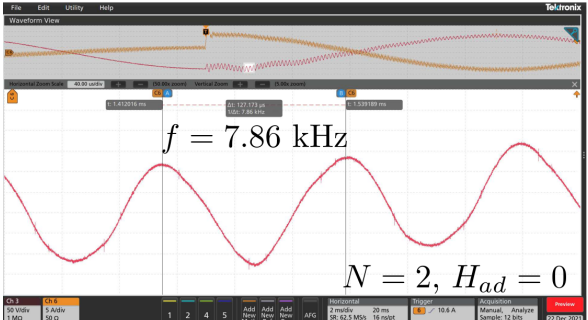

j)
$Z_{g, 2}: f_{\text {res }}=18.6 \mathrm{kHz}, Z_{c}=48 \%$

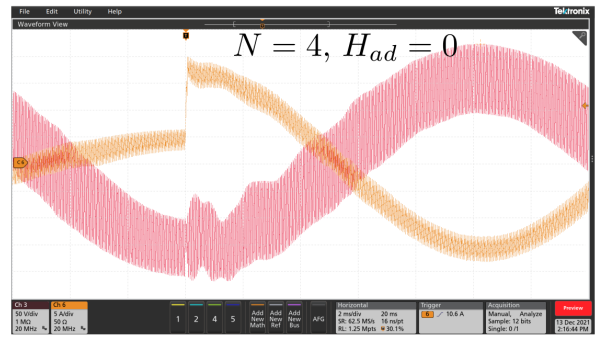

b)

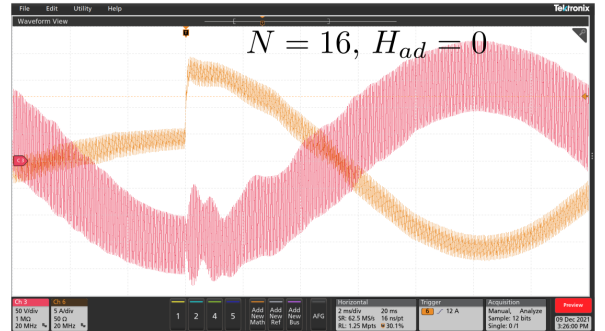

e)

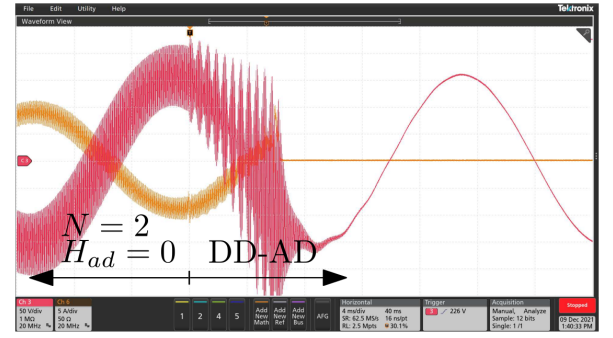

h)

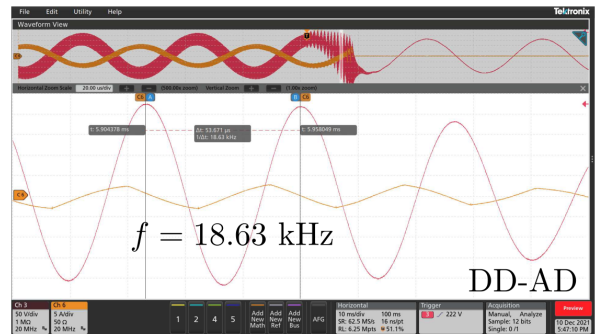

k)
$Z_{g, 3}: f_{\text {res }}=28.4 \mathrm{kHz}, Z_{c}=73 \%$

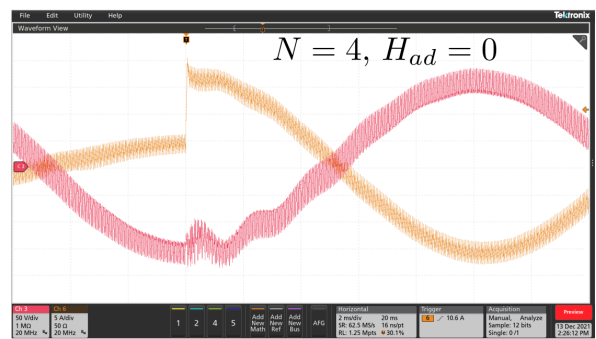

c)

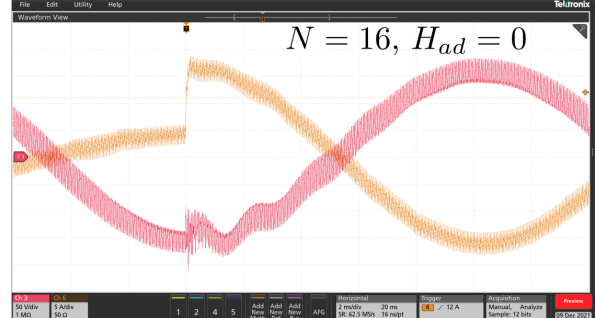

f)

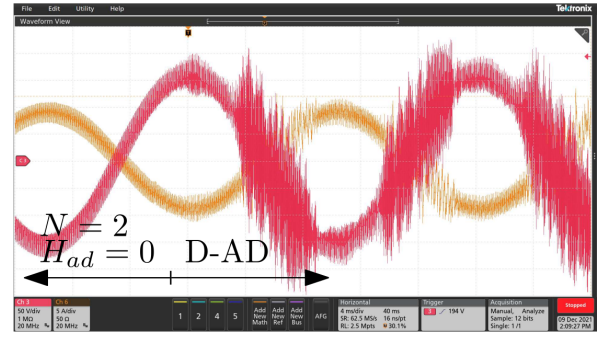

i)

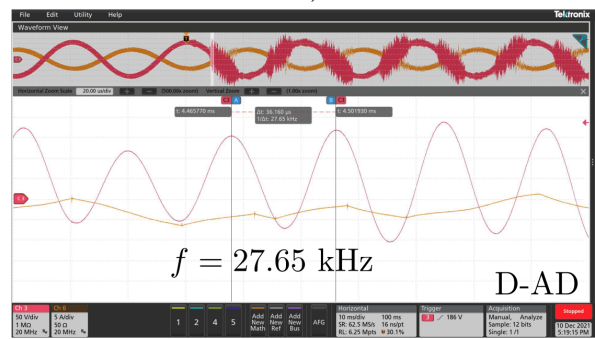

l)

Fig. 11: Experimental demonstration of passivity implications in grid-connected scenarios. Orange line shows $i_{L}$ and red line shows $v_{p c c}$ that has variable ripple depending on $C_{g}$. Each column features results for different tested grid impedances $Z_{g}$. Grid impedances are formed as $L C$ anti-resonant circuits and feature certain passive damping. Results are shown as transient responses to step reference change for: a), b), c) MS-PWM with N = 4; d), e), f) MS-PWM with $N=16$; g) DS-PWM without AD with non-passive zone of $Y_{i}$ coinciding with $Z_{g, 1}$ anti-resonance. Operation features strong oscillations at $f_{r e s}$ but passive grid damping prevents instability; h) Operation change from DS-PWM without AD to DD-AD. Due to weakly-damped $Z_{g, 2}$ anti-resonance, coinciding with the non-passive zone of $Y_{i}$, instability occurs and protection is triggered; i) Operation change from DS-PWM without AD to D-AD. Due to weakly-damped $Z_{g, 3}$ anti-resonance, coinciding with the non-passive zone of $Y_{i}$, instability occurs; j), k), l) zoomed waveforms from the row above, showing oscillation frequencies.

component and its harmonics are removed, so that distortion does not impact variance calculations. Input noise is estimated in open-loop condition using sampled data from the control platform with $N=N_{a d}=2$. Estimated input variances are equal to $\sigma_{i}^{2}=5 \cdot 10^{-4} \mathrm{~A}^{2}$ and $\sigma_{v}^{2}=80 \cdot 10^{-3} \mathrm{~V}^{2}$. As a benchmark, simulations are implemented in Simulink using PLECS Blockset. White noise is injected, with variances corresponding to measured $\sigma_{i}^{2}$ and $\sigma_{v}^{2}$.

Measured output variances are shown in Fig. 12, relative to the simulation results for DS-PWM without AD. Noise attenuation depends on the magnitude response of the transfer function from sensed noise to the output $i_{L}$ [40]. For DD-
$\mathrm{AD}$, results show almost the same output noise as for the case of DS-PWM without AD. As these cases feature the same magnitude response from $n_{i}$ to $i_{L}$, it is concluded that the voltage noise propagation is completely masked by the stronger current noise propagation. This outcome is a result of a relatively low derivative gain $k_{a d}$ (9). Intuitively, for setups that feature much higher ratio $\frac{\sigma_{v}^{2}}{\sigma_{i}^{2}}$, higher noise sensitivity of $\mathrm{AD}$ methods is expected. For ${ }^{i} \mathrm{D}-\mathrm{AD}$, results in Fig. 12 show approximately twice increased output noise power. As shown in [40], oversampled derivative followed by decimation without anti-aliasing filtering amplifies the noise power. Again, 


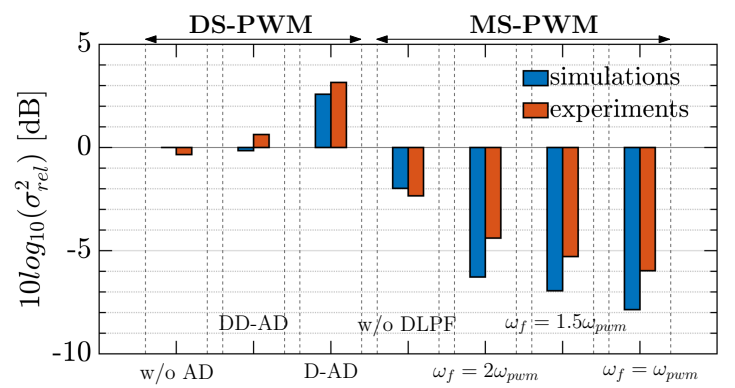

Fig. 12: Results of noise measurements: $L=2.5 \mathrm{mH}, \alpha=0.1$. Values are given relative to simulation results for DS-PWM without AD.

it is expected that this sensitivity depends on values of $\frac{\sigma_{v}^{2}}{\sigma_{i}^{2}}$ and $N_{a d}$ [40]. Second mechanism, important for MS-PWM, is determined by the sampling frequency $f_{s}$, as white noise content is spread up to the NF [40]. For linear systems and white noise, changing the oversampling factor from $N_{1}$ to $N_{2}$ results in a change of the white noise power, in the observed frequency window, equal to $\frac{\sigma_{N_{2}}^{2}}{\sigma_{N_{1}}^{2}}=\frac{N_{1}}{N_{2}}$ [40]. As analyzed in [40], for current loops without derivative gain, for $N>2$ noise power remains nearly constant due to aliasing caused by DPWM re-sampling effects [40]; hence, additional high-frequency digital filtering is needed to enable strong noise suppression in the lower frequency range. For this paper, three digital low-pass filters (DLPFs) $G_{f b}(z)$ are tested to observe their impact on noise attenuation. DLPFs are implemented as first-order filters with cut-off frequencies $\omega_{f}=\{2,1.5,1\} \cdot \omega_{\text {pwm }}$ and are discretized using the Bilinear transform. It is important to notice that these DLPFs do not directly attenuate noise power in the observed frequency window. The design goal is to suppress noise power above $f_{\text {pwm }}$ with as little impact on dynamic performance ${ }^{4}$. DLPFs with lower cut-off frequencies bring more filtering at $f>f_{\text {pwm }}$; hence, corresponding results are expected to approach the value determined by $10 \log _{10}\left(\frac{\sigma_{N}^{2}}{\sigma_{2}^{2}}\right)=10 \log _{10}\left(\frac{2}{16}\right) \approx-9 \mathrm{~dB}$. This trend is confirmed by results in Fig. 12. Even without DLPF, noise power is reduced compared to DS-PWM. This is due to a higher phase margin, which brings additional filtering by the magnitude response from $n_{i}$ to $i_{L}$. Note that all experimental measurements are in a good match with simulations.

Modeling of decimation impacts on noise propagation is left for future work, due to paper length limitations. The main conclusion of this section is that implementing AD does not necessarily result in an excessive noise sensitivity. Still, MSPWM offers additional noise suppression.

\section{CONCLUSIONS}

This paper has provided a comparison between different strategies used to render the input admittance of grid-

\footnotetext{
${ }^{4}$ In order to verify that implemented DLPFs do not strongly deteriorate passivity properties, admittances are measured for tested DLPFs and only a small impact is seen. Peaks of negative conductance, measured for $\alpha=0.2$ for compatibility with results in Table II, are $\{-0.25,-0.17\} \%$ for $L=$ $\{1.5,2.5\} \mathrm{mH}$ at $28 \mathrm{kHz}$.
}

connected VSCs passive. MS-PWM is compared against typically used AD methods. It is shown that MS-PWM outperforms $\mathrm{AD}$ in terms of effectiveness, as $\mathrm{AD}$ brings negative impact at high frequencies. VSC is connected to a grid with different anti-resonances to show that only MS-PWM enables stability for all cases. Additionally, noise propagation results have shown that $\mathrm{AD}$ tactics are not extremely sensitive to measurement noise. Still, MS-PWM outperforms AD in this respect as well. The study shown in this paper further points out that MS-PWM is a very viable solution for achieving high bandwidths, while guaranteeing stability of current-controlled grid-connected VSCs.

\section{REFERENCES}

[1] F. Blaabjerg, R. Teodorescu, M. Liserre, and A. Timbus, "Overview of control and grid synchronization for distributed power generation systems," IEEE Transactions on Industrial Electronics, vol. 53, no. 5, pp. 1398-1409, 2006

[2] D. Boroyevich, I. Cvetkovic, R. Burgos, and D. Dong, "Intergrid: A future electronic energy network?" IEEE Journal of Emerging and Selected Topics in Power Electronics, vol. 1, no. 3, pp. 127-138, 2013.

[3] X. Wang and F. Blaabjerg, "Harmonic stability in power electronic-based power systems: Concept, modeling, and analysis," IEEE Transactions on Smart Grid, vol. 10, no. 3, pp. 2858-2870, 2019.

[4] L. Harnefors, A. G. Yepes, A. Vidal, and J. Doval-Gandoy, "Passivitybased controller design of grid-connected vscs for prevention of electrical resonance instability," IEEE Transactions on Industrial Electronics. vol. 62 , no. 2, pp. 702-710, 2015.

[5] S. Buso and P. Mattavelli, "Digital control in power electronics, 2nd edition," Synthesis Lectures on Power Electronics, Morgan \& Claypool Publishers, USA, 2015.

[6] "Railway Applications-Power Supply and Rolling Stock-Technical Criteria for the Coordination Between Power Supply (Substation) and Rolling Stock to Achieve Interoperability, CENELEC Std. EN50388, 2012."

[7] L. Harnefors, X. Wang, A. G. Yepes, and F. Blaabjerg, "Passivitybased stability assessment of grid-connected vscs-an overview," IEEE Journal of Emerging and Selected Topics in Power Electronics, vol. 4, no. 1, pp. 116-125, 2016.

[8] L. Harnefors, R. Finger, X. Wang, H. Bai, and F. Blaabjerg, "Vsc input-admittance modeling and analysis above the nyquist frequency for passivity-based stability assessment," IEEE Transactions on Industrial Electronics, vol. 64, no. 8, pp. 6362-6370, 2017.

[9] X. Wang, F. Blaabjerg, and P. C. Loh, "Passivity-based stability analysis and damping injection for multiparalleled vscs with lcl filters," IEEE Transactions on Power Electronics, vol. 32, no. 11, pp. 8922-8935, 2017.

[10] E. Rodriguez-Diaz, F. D. Freijedo, J. M. Guerrero, J.-A. MarreroSosa, and D. Dujic, "Input-admittance passivity compliance for gridconnected converters with an lcl filter," IEEE Transactions on Industrial Electronics, vol. 66, no. 2, pp. 1089-1097, 2019.

[11] H. Yu, M. A. Awal, H. Tu, Y. Du, S. Lukic, and I. Husain, "Passivityoriented discrete-time voltage controller design for grid-forming inverters," in 2019 IEEE Energy Conversion Congress and Exposition (ECCE), 2019, pp. 469-475.

[12] H. Wu and X. Wang, "Virtual-flux-based passivation of current control for grid-connected vscs," IEEE Transactions on Power Electronics, vol. 35, no. 12, pp. 12673-12 677, 2020.

[13] M. A. Awal, W. Yu, and I. Husain, "Passivity-based predictive-resonant current control for resonance damping in lcl-equipped vscs," IEEE Transactions on Industry Applications, vol. 56, no. 2, pp. 1702-1713, 2020.

[14] A. Akhavan, H. R. Mohammadi, J. C. Vasquez, and J. M. Guerrero, "Passivity-based design of plug-and-play current-controlled gridconnected inverters," IEEE Transactions on Power Electronics, vol. 35, no. 2, pp. 2135-2150, 2020.

[15] J. Serrano-Delgado, S. Cobreces, M. Rizo, and E. J. Bueno, "Low-order passivity-based robust current control design for grid-tied vscs," IEEE Transactions on Power Electronics, vol. 36, no. 10, pp. 11 886-11 899, 2021. 
[16] Z. Yang, C. Shah, T. Chen, J. Teichrib, and R. W. De Doncker, "Virtual damping control design of three-phase grid-tied pv inverters for passivity enhancement," IEEE Transactions on Power Electronics, vol. 36, no. 6, pp. 6251-6264, 2021.

[17] M. A. Awal, L. D. Flora, and I. Husain, "Observer based generalized active damping for voltage source converters with lcl filters," IEEE Transactions on Power Electronics, vol. 37, no. 1, pp. 125-136, 2022.

[18] J. Zhao, C. Xie, K. Li, J. Zou, and J. M. Guerrero, "Passivity-oriented design of lcl-type grid-connected inverters with luenberger observerbased active damping," IEEE Transactions on Power Electronics, vol. 37, no. 3 , pp. 2625-2635, 2022.

[19] F. Hans, W. Schumacher, S.-F. Chou, and X. Wang, "Passivation of current-controlled grid-connected vscs using passivity indices," IEEE Transactions on Industrial Electronics, vol. 66, no. 11, pp. 8971-8980, 2019.

[20] C. Xie, K. Li, J. Zou, and J. M. Guerrero, "Passivity-based stabilization of lcl-type grid-connected inverters via a general admittance model," IEEE Transactions on Power Electronics, vol. 35, no. 6, pp. 6636-6648, 2020.

[21] Y. Liao, X. Wang, and F. Blaabjerg, "Passivity-based analysis and design of linear voltage controllers for voltage-source converters," IEEE Open Journal of the Industrial Electronics Society, vol. 1, pp. 114-126, 2020.

[22] S. Li and H. Lin, "A capacitor-current-feedback positive active damping control strategy for lcl-type grid-connected inverter to achieve high robustness," IEEE Transactions on Power Electronics, pp. 1-1, 2021.

[23] X. Wang, Y. He, D. Pan, H. Zhang, Y. Ma, and X. Ruan, "Passivity enhancement for lcl-filtered inverter with grid current control and capacitor current active damping," IEEE Transactions on Power Electronics, vol. 37, no. 4, pp. 3801-3812, 2022.

[24] R. Middlebrook, "Input filter considerations in design and application of switching regulators." 1976.

[25] J. Sun, "Impedance-based stability criterion for grid-connected inverters," IEEE Transactions on Power Electronics, vol. 26, no. 11, pp. 30753078, 2011 .

[26] B. Wen, D. Dong, D. Boroyevich, R. Burgos, P. Mattavelli, and Z. Shen, "Impedance-based analysis of grid-synchronization stability for threephase paralleled converters," IEEE Transactions on Power Electronics, vol. 31, no. 1, pp. 26-38, 2016.

[27] X. Wang, L. Harnefors, and F. Blaabjerg, "Unified impedance model of grid-connected voltage-source converters," IEEE Transactions on Power Electronics, vol. 33, no. 2, pp. 1775-1787, 2018.

[28] F. D. Freijedo, M. Ferrer, and D. Dujic, "Multivariable high-frequency input-admittance of grid-connected converters: Modeling, validation, and implications on stability," IEEE Transactions on Industrial Electronics, vol. 66, no. 8, pp. 6505-6515, 2019.

[29] V. Pirsto, J. Kukkola, M. Hinkkanen, and L. Harnefors, "Intersample modeling of the converter output admittance," IEEE Transactions on Industrial Electronics, vol. 68, no. 11, pp. 11348-11358, 2021.

[30] J. Dannehl, M. Liserre, and F. W. Fuchs, "Filter-based active damping of voltage source converters with lcl filter," IEEE Transactions on Industrial Electronics, vol. 58, no. 8, pp. 3623-3633, 2011.

[31] G. Walker, "Digitally-implemented naturally sampled pwm suitable for multilevel converter control," IEEE Transactions on Power Electronics, vol. 18, no. 6, pp. 1322-1329, 2003.

[32] L. Corradini and P. Mattavelli, "Modeling of multisampled pulse width modulators for digitally controlled dc-dc converters," IEEE Transactions on Power Electronics, vol. 23, no. 4, pp. 1839-1847, 2008.

[37] S. He, D. Zhou, X. Wang, and F. Blaabjerg, "Aliasing suppression of multi-sampled current controlled lcl-filtered inverters," IEEE Journal of Emerging and Selected Topics in Power Electronics, pp. 1-1, 2021
[33] L. Corradini, W. Stefanutti, and P. Mattavelli, "Analysis of multisampled current control for active filters," IEEE Transactions on Industry Applications, vol. 44, no. 6, pp. 1785-1794, 2008.

[34] X. Zhang, P. Chen, C. Yu, F. Li, H. T. Do, and R. Cao, "Study of a current control strategy based on multisampling for high-power gridconnected inverters with an lcl filter," IEEE Transactions on Power Electronics, vol. 32, no. 7, pp. 5023-5034, July 2017.

[35] J. Ma, X. Wang, F. Blaabjerg, W. Song, S. Wang, and T. Liu, "Multisampling method for single-phase grid-connected cascaded h-bridge inverters," IEEE Transactions on Industrial Electronics, vol. 67, no. 10, pp. 8322-8334, 2020.

[36] Z. Zhou, J. Wang, Z. Liu, and J. Liu, "Accurate prediction of vertical crossings for multi-sampled digital-controlled buck converters," in 2020 IEEE Applied Power Electronics Conference and Exposition (APEC), 2020, pp. 292-298.

[38] I. Z. Petric, P. Mattavelli, and S. Buso, "A jitter amplification phenomenon in multisampled digital control of power converters," IEEE Transactions on Power Electronics, vol. 36, no. 8, pp. 8685-8695, 2021.

[39] I. Petric, R. Cvetanovic, P. Mattavelli, S. Buso, and S. Vukosavic, "Analysis and dsp implementation of multi-sampled three-phase current controllers," in 2021 21st International Symposium on Power Electronics (Ee), 2021, pp. 1-7.

[40] I. Z. Petric, P. Mattavelli, and S. Buso, "Feedback noise propagation in multisampled dc-dc power electronic converters," IEEE Transactions on Power Electronics, vol. 37, no. 1, pp. 150-161, 2022.

[41] _ , "Investigation of nonlinearities introduced by multi-sampled pulsewidth modulators," IEEE Transactions on Power Electronics, vol. 37, no. 3, pp. 2538-2550, 2022.

[42] I. Petric, P. Mattavelli, and S. Buso, "Multi-sampled grid-connected vscs: A path towards inherent admittance passivity," IEEE Transactions on Power Electronics, pp. 1-1, 2022.

[43] H. d. T. Mouton, S. M. Cox, B. McGrath, L. Risbo, and B. Putzeys, "Small-signal analysis of naturally-sampled single-edge pwm control loops," IEEE Transactions on Power Electronics, vol. 33, no. 1, pp. 51-64, 2018.

[44] D. Van de Sype, K. De Gusseme, A. Van den Bossche, and J. Melkebeek, "Small-signal laplace-domain analysis of uniformly-sampled pulsewidth modulators," in 2004 IEEE 35th Annual Power Electronics Specialists Conference (IEEE Cat. No.04CH37551), vol. 6, 2004, pp. 4292-4298 Vol.6.

[45] R. Cvetanovic, I. Petric, P. Mattavelli, and S. Buso, "Accurate high frequency modelling of the input admittance of pwm grid-connected vscs," under revision in IEEE Transactions on Power Electronics, uploaded to TechRxiv.

[46] R. Gupta, A. Ghosh, and A. Joshi, "Characteristic analysis for multisampled digital implementation of fixed-switching-frequency closed-loop modulation of voltage-source inverter," IEEE Transactions on Industrial Electronics, vol. 56, no. 7, pp. 2382-2392, 2009.

[47] P. Mattavelli, F. Polo, F. Dal Lago, and S. Saggini, "Analysis of controldelay reduction for the improvement of ups voltage-loop bandwidth," IEEE Transactions on Industrial Electronics, vol. 55, no. 8, pp. 29032911, 2008.

[48] D. Yang, X. Ruan, and H. Wu, "A real-time computation method with dual sampling mode to improve the current control performance of the lcl-type grid-connected inverter," IEEE Transactions on Industrial Electronics, vol. 62, no. 7, pp. 4563-4572, 2015. 